\title{
Laparoscopic interval surgery for stage 4 primary fallopian tube carcinoma-a case report
}

\author{
Thumuluru Kavitha Madhuri • Anil Tailor • \\ Simon Butler-Manuel
}

Received: 21 June 2009 / Accepted: 9 July 2009 /Published online: 1 August 2009

(C) Springer-Verlag 2009

\begin{abstract}
Primary fallopian tube carcinoma (PFTC) is an uncommon malignancy of the female genital tract. Rationale for treatment of PFTC is similar to epithelial ovarian cancer (EOC) due to pathological similarities and pattern of disease spread. Surgical cytoreduction of advanced stage or large volume disease has been by laparotomy and debulking. We present the first case of laparoscopic interval surgery (IS) for stage IVb PFTC minimising peri-operative morbidity with no delay in resuming chemotherapy. This case demonstrates that there exists a role for laparoscopic surgery in selected patients which should be explored.
\end{abstract}

Keywords Primary fallopian tube carcinoma - Epithelial ovarian cancer - Interval surgery Cytoreduction . Laparoscopy

\section{Background}

Primary fallopian tube carcinoma (PFTC) is one of the least common malignancies of the female genital tract accounting for less than $1 \%$ of all gynaecological tumours worldwide. Traditionally, advanced stage disease is treated by laparotomy and debulking followed by chemotherapy

This case discusses the possibility of exploring the option of laparoscopic surgery in selected patients with ovarian, primary peritoneal or fallopian tube malignancies which improves patient quality of life.

T. K. Madhuri $(\square) \cdot$ A. Tailor $\cdot$ S. Butler-Manuel

Department of Gynaecological Oncology,

The Royal Surrey County Hospital,

LEVEL B, GOPD, Egerton Road,

Guildford, Surrey GU2 7XX, UK

e-mail: docmadhuri231@doctors.org.uk following the principles of management of epithelial ovarian cancer (EOC).

Neo-adjuvant chemotherapy followed by interval debulking surgery (IDS) is increasingly seen as a valid treatment option for advanced stage EOC and PFTC and its use is supported by the evidence from the recent EORTCGCG/NCIC-CTG randomised trial [1]. This generally involves open debulking to attain optimal cytoreduction.

We present the first reported use of laparoscopic IS in a patient with stage 4 PFTC enabling early recovery and quicker recommencement of adjuvant chemotherapy.

\section{Case summary}

A 62-year-old multiparous lady was referred with 6-week history of loss of weight and appetite. Examination revealed a distended abdomen. Breast examination was normal. Her medical history was otherwise unremarkable except for a strong family history of breast malignancy. CT scan showed peritoneal disease, normal looking ovaries but a solid $4 \times 3-\mathrm{cm}$ mass near the left uterine fundus, enlarged bilateral pelvic and para-aortic (PA) nodes (see Figs. 1, 2, 3 and 4). Her CA-125 was markedly elevated at 4,559 kU/L (normal range $0-35$ ). Other investigations (mammography and oesophagogastroduodenoscopy) were normal and a diagnostic laparoscopy was performed. This showed a normal peritoneum and pelvis except for a dilated left tube. Laparoscopic biopsies were taken from both tubes and ovaries and the pelvic peritoneum. Histology of the left tube biopsy was reported as poorly differentiated adenocarcinoma consisted with a fallopian tube malignancy. Immunohistochemistry (IHC) was positive for cytokeratin (CK) 7 and negative for CK20 consistent with an occult gynaecological primary. Biopsies from the right fallopian 


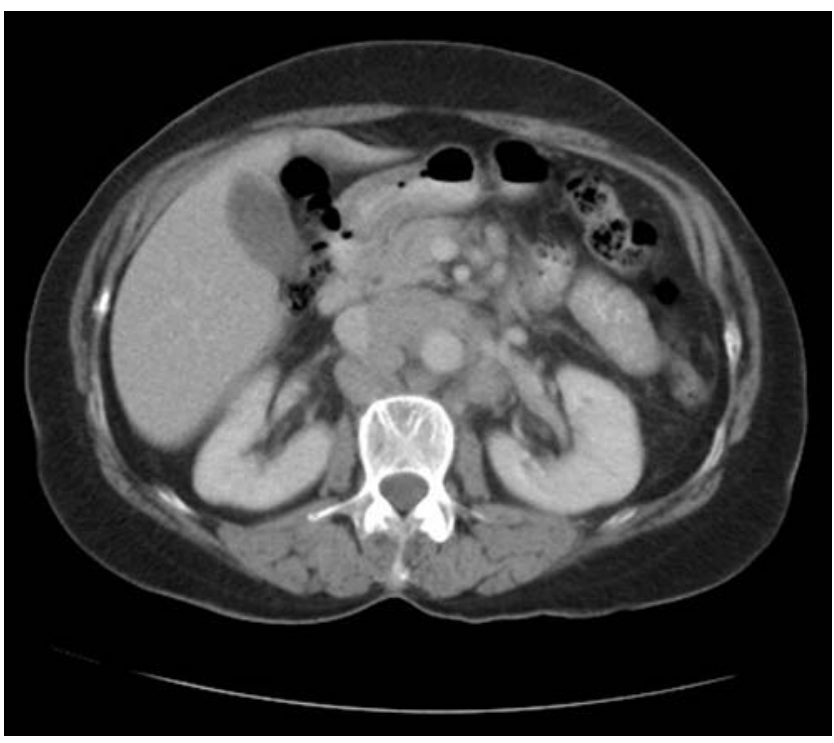

Fig. 1 CT scan showing enlarged para-aortic lymph nodes

tube and both ovaries were normal. While investigations were ongoing, she developed swelling in the anterior triangle of the left side of the neck with painful neck movements. Examination revealed enlarged supraclavicular nodes (SCL) and histology from a core biopsy taken from one of the SCL nodes was consistent with a fallopian tube primary and a diagnosis of FIGO stage IVb PFTC was made.

Following discussion at the gynae-oncology multidisciplinary team meeting (MDT), neo-adjuvant chemotherapy was recommended and following consultation with the patient, chemotherapy was commenced with 3-weekly carboplatin and paclitaxel. CT scan was repeated following cycle 4 which showed an excellent response with complete resolution of the pelvic and PA nodes, reduction in the size of the mass in the anterior triangle of neck and no evidence

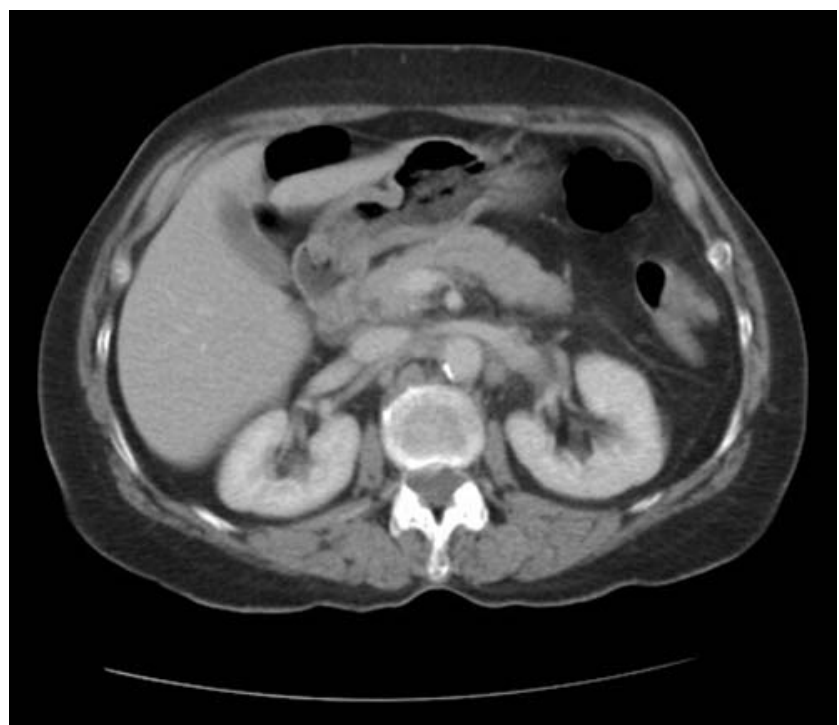

Fig. 2 Interval CT scan after cycle 4

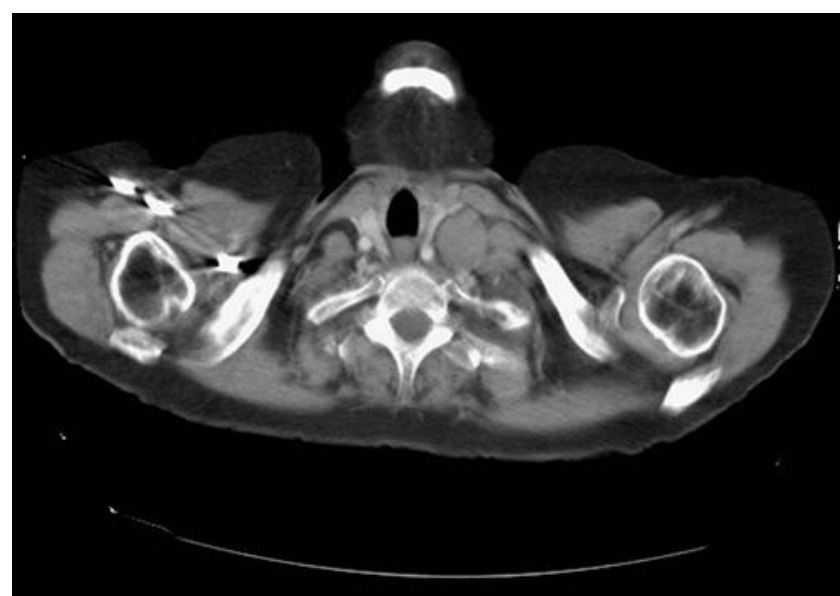

Fig. 3 CT scan showing enlarged left supraclavicular nodes consistent with metastatic disease

of abdominal disease. Her CA-125 had dropped to 62 . Her case was rediscussed at the MDT and the option of IS debated including the possible merits of PA node dissection. The patient's wishes were taken into consideration as she was keen to avoid major surgery if possible. It was decided to perform a further laparoscopic assessment and cytoreductive surgery. The MDT decided that PA node dissection was not worthwhile as these nodes had greatly diminished in size and resection of these would not add greatly to her outcome and prognosis in view of her enlarged SCL nodal disease at presentation.

Successful laparoscopic surgery was carried out 20 days after her fourth cycle of chemotherapy and included a laparoscopic total hysterectomy, bilateral salpingooopherectomy with stripping of the surrounding pelvic peritoneum and supracolic omentectomy. The surgery was carried out using Harmonic ACE ${ }^{\mathrm{TM}}$. At laparoscopy, there was no ascites, uterus was of normal size and mobile with a small volume left adnexal mass present. There was no obvious

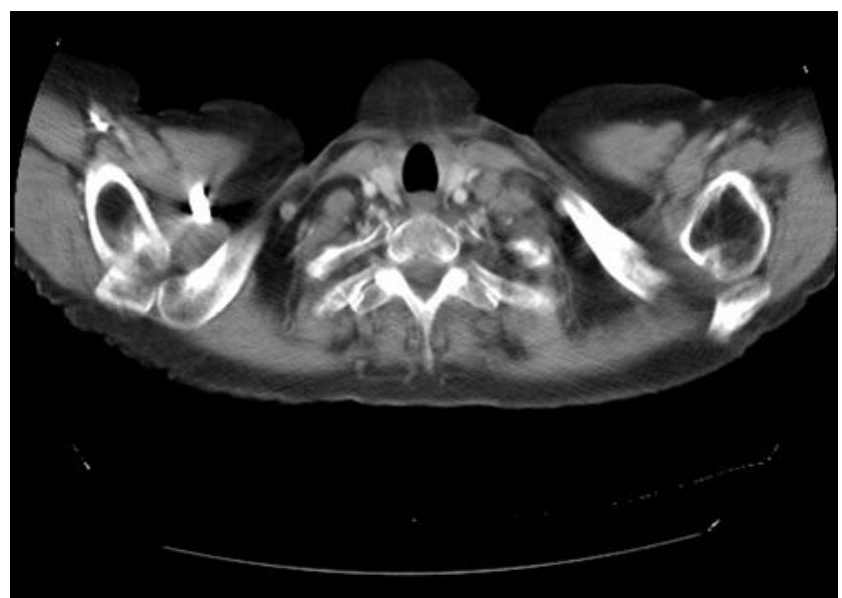

Fig. 4 Interval CT after 4 cycles showing reduction in size of lymph nodes 
uterine serosal disease or omental nodularity but the pelvic peritoneum in the left adnexum appeared abnormal and was widely excised en bloc together with the uterus, tubes and ovaries. The liver and diaphragm appeared normal. All visible disease was excised but no node dissection was performed. Blood loss was $200 \mathrm{~mL}$ and she made a routine postoperative recovery and was discharged the following day. The histology showed carcinoma within the full thickness of the left fallopian tube and paratubal tissue extending into the serosa at the left cornua of the uterus. Left ovary, right tube, ovary, parametria, uterus, cervix and omentum were free of disease. Excision margins were clear of disease. She restarted her chemotherapy 2 days post-operatively with no delay in her treatment schedule. She is 20 months post treatment and disease free.

\section{Discussion}

PFTC was first reported by Orthmann in 1888 [2]. PFTC is a very rare malignancy and accounts for less than $2 \%$ of malignancies diagnosed among women worldwide with 1,200 cases reported worldwide in the literature [3]. Early stage disease may present with vaginal discharge or postmenopausal bleeding. PFTC may also be mistaken for EOC at initial diagnosis and during histological examination of staging biopsies as the histological and biological features of both are similar (papillary serous adenocarcinoma) [4]. Population-based cancer registries in the USA show 3,051 PFTC cases diagnosed during 1998 to 2003 and the incidence is reported as 0.41 per 100,000 women [4].

Clear histological criteria for differentiating PFTC from other gynaecological malignancies was described in 1950 and later modified by Sedlis in 1961 and 1978 which stated the main tumour must arise from the endosalpinx with smaller or no tumour in the ovaries and endometrium which is met by our case [5]. As the histology and patterns of spread of disease are similar, treatment of PFTC shadows the treatment of EOC and advocates extensive cytoreductive surgery followed by chemotherapy [6].

Minimally invasive surgery is recommended in early stage disease for staging or re-staging following an unexpected diagnosis of malignancy and in advanced disease to assess extent of disease and resectability prior to proceeding to a laparotomy [7]. Laparoscopic surgery is not generally recommended for majority of ovarian cancers due to limitations of dealing with large tumour volumes and the potential risk of dissemination of tumour cells by the pneumoperitoneum. Concerns related to laparoscopic surgery also include incomplete staging by sub-optimal assessment of peritoneal/mesenteric/nodal disease, tumour spillage and port site metastasis [8].

We routinely advocate node dissection (pelvic and paraaortic) for patients with EOC for both staging and cytoreductive purposes. PA node dissection was discussed following chemotherapy but this patient already had confirmed stage IVb disease due to positive SCL node biopsy. As SCL neck dissection was not going to be entertained and she had shown complete resolution of her pelvic and PA nodes, laparoscopic excision was offered to reduce surgical morbidity and early resumption of chemotherapy. Laparoscopic surgery has the advantage of less morbidity, shorter recovery and early restarting of chemotherapy [9]. This case illustrates the benefits of an initial staging laparoscopy in the management of these cases which influenced subsequent decision making.

We recognise that large studies with greater number of patients to assess the feasibility, safety and success of laparoscopic surgery for stage IV PFTC or stage IV EOC will be difficult to achieve due to low incidence of these tumours.

In conclusion, we believe there is a role for laparoscopic cytoreduction in selected patients diagnosed with stage IV PFTC or stage IV EOC following good response to neoadjuvant chemotherapy.

Conflict of interest There is no actual or potential conflict of interest in relation to this article.

\section{References}

1. EORTC-GCG/NCIC-CTG Randomised Trial Comparing Primary Debulking Surgery With Neoadjuvant Chemotherapy in Stage IIIC-IV Ovarian, Fallopian Tube and Peritoneal Cancer (OVCA). Vergote et al. Proceedings from 12th International Gynecologic Cancer Society-IGCS

2. Hanton EM, Malkasian GD Jr, Dahlin DC, Pratt JH (1996) Primary carcinoma of the fallopian tube. Am J Obstet Gynecol 94:832-839

3. Pectasides D, Pectasides E, Economopoulos T (2006) Fallopian tube carcinoma: a review the oncologist 11:902-912

4. Stewart SL, Wike JM, Foster SL, Michaud F (2007) The incidence of primary fallopian tube cancer in the United States. Gynecol Oncol 107(3):392-397

5. Clayton NL, Jaaback KS, Hirschowitz L (2005) Primary fallopian tube carcinoma - the experience of a UK cancer centre and a review of the literature. J Obstet Gynaecol 25(7):694-702

6. Moore $\mathrm{KN}$ et al (2007) Serous fallopian tube carcinoma: a retrospective, multi-institutional case-control comparison to serous adenocarcinoma of the ovary. Gynecol Oncol 107(3):398-403

7. Schlaerth AC, Abu-Rustum NR (2006) Role of minimally invasive surgery in gynecologic cancers. The Oncologist 11:895-901

8. Ghezzi $\mathrm{F}$ et al (2007) Laparoscopy versus laparotomy for the surgical management of apparent early stage ovarian cancer. Gynecol Oncol 105(2):409-413

9. Querleu D, Leblanc E, Ferron G, Narducci F (2006) Laparoscopic surgery in gynaecological oncology. Eur J Surg Oncol 32(8):853858

Harmonic ACE ${ }^{\mathrm{TM}}$ is a trademark of Ethicon Endo-Surgery. 